\section{Intense prolonged hypocalcemia and hyperparathyroidism after Denosumab in hemodialysis patients in response to the published article}

\section{Clinical case}

38 years old woman in hemodialysis since 2001 by lupus nephritis, and other backgrounds such as severe artery antiphospholipid syndrome, chronic hepatitis $\mathrm{C}$ treated with, and intestinal angiodysplasia. After receiving several cycles of cyclophosphamide and chronic steroid therapy from 12 years of age, has osteoporosis at high risk of fracture and bone loss in trabecular bone compact (total Ts -2.1, -1.2 Zs), for which she was treated with Risedronate from January 2009 to April 2012, when densitometry (BMD) is repeated by increasing osteoporosis intensity ( $\mathrm{T}-2.5,-2.4 \mathrm{Zs}$ ) and then deciding to change Denosumab. Her bone mineral metabolism has always been satisfactory with good control figures calcium, phosphorus and mild hyperparathyroidism generally well controlled with low doses of paricalcitol and unspecified calcimimetic. After the first and second dose of Denosumab, it presents mild hypocalcemia recovered easily and rapidly FIGURE 1.

To January 2014 does not receive the third Denosumab dose for different reasons (severe lupus flare, tunnelled catheter infection, gastrointestinal bleeding) presenting after administration, severe hypocalcemia, hypophosphoremia poner entre paréntesis and severe hyperparathyroidism she had never previously presented. The figures magnesium, although descended, did not change significantly compared to the months-years previous, and did so the Alkaline Phosphatase who had always maintained in the normal range, with no signs of liver disease (progressive increase from 44 IU / L before the first dose to 187 IU / L after the third dose) FIGURE 1 AND 2.

Coinciding with the administration of the 3rd dose, poner solo patient was treated with calcium acetate, magnesium carbonate, calcifediol, acenocoumarol, mycophenolic acid, folic acid, vitamin B and C, esomeprazole, mirtazapine, lormetazepam and Octeotride for intestinal severe angyodisplasia plus variable dose of intravenous iron and darbepoetin.

IV calcium and oral magnesium carbonate later (eliminate "and oral contribution beginning") with magnesium carbonate later. Moreover, dialysis bath with positive balance in each of the four weekly hemodialysis sessions, in which vitamin D3 1.25 IV administered. However, the figures calcium, phosphorus and PTH body took 3 to 4 months to recover. Currently, patient remains asymptomatic in this regard.

\section{Discussion}

Denosumab is a human monoclonal IgG2 antibody indicated in postmenopausal osteoporosis [1] or prostatic neoplasia. The

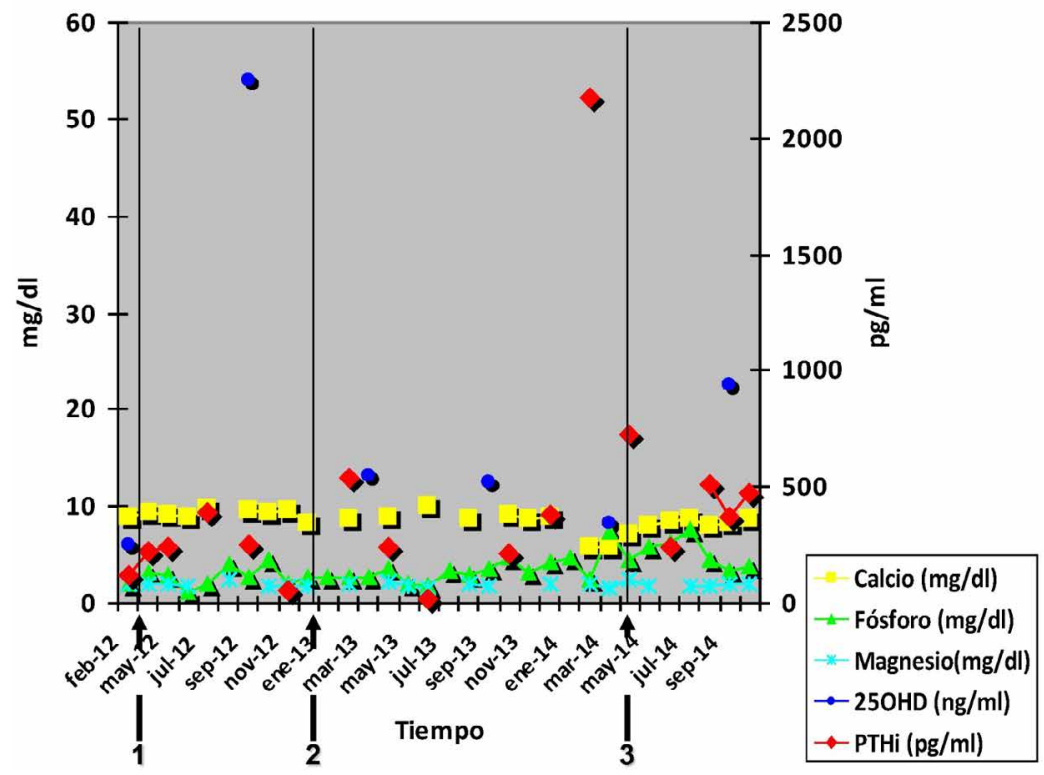

Figure 1. Laboratory progression of Denosumab, after 3 doses. Hypocalcaemia is observed after the second and third doses, PTH increased after each of the administrations but more striking after the last. Arrows 1,2,3 administrations of drug over time.
Martin-Gomez MA*, Martinez de la Plata E, Hidalgo Rico MA, Palacios Gomez ME \& Garcia-Marcos S

Units Nephrology and Neumologia, Public Enterprise Hospital de Poniente, Almeria, Spain

*Author for correspondence:

Tel.: 677081239

doritamg@gmail.com 

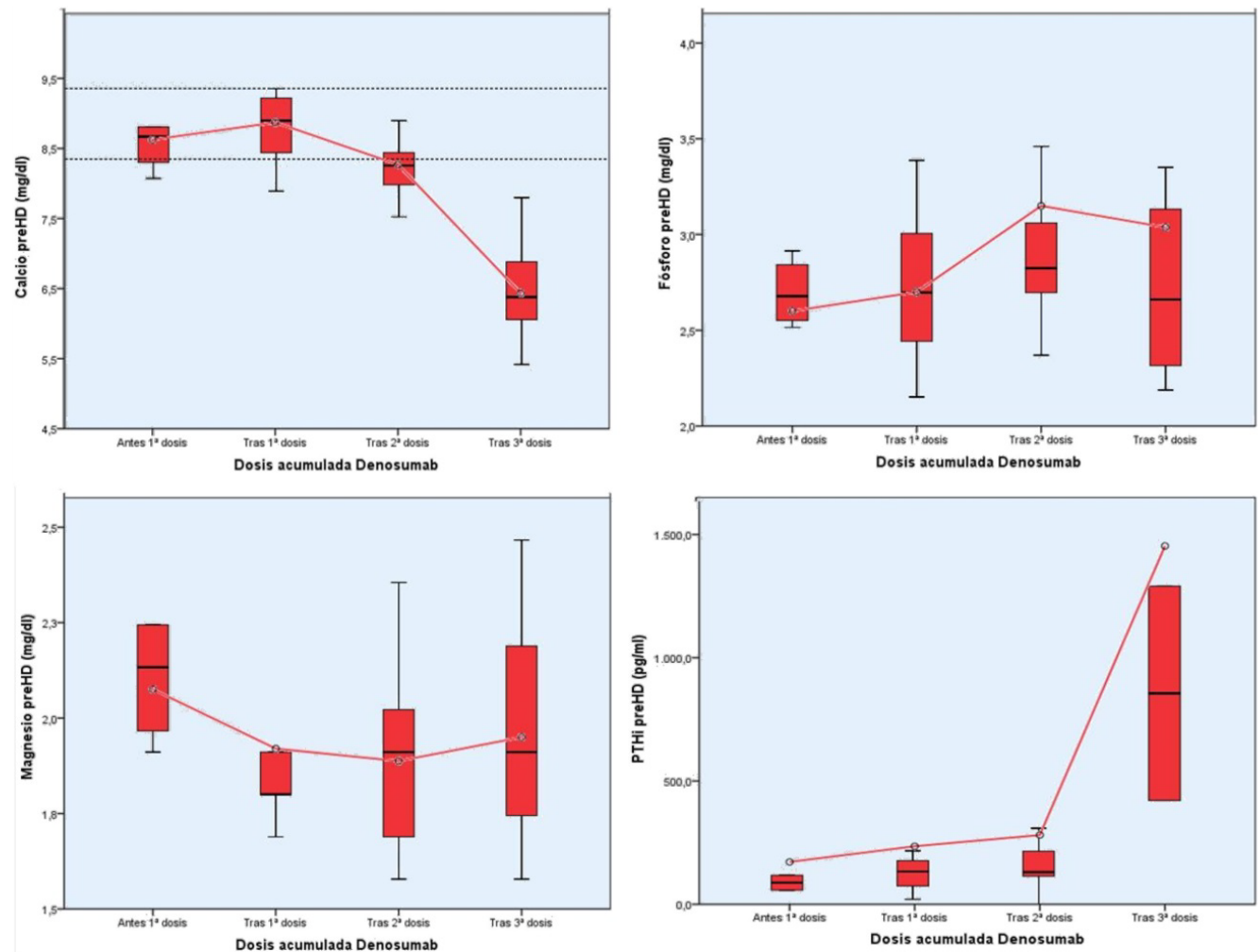

Figure 2. Comparative analytical evolution after 3 doses of Denosumab. The medians of the 4 episodes are compared by Kruskal-Wallis statistics, being significant decrease occurred calico corrected after 3 doses of the drug, with respect to the previous $(p=0.015)$. Although the rise of PTH is striking, with no significance may be due to the shortage of shows to do. The solid line corresponds to the union of the means at different times

indication in osteopenia / osteoporosis for other reasons is not yet approved, but described work that could support its use in patients with advanced chronic kidney disease [2-4] due to the improvement observed in bone mineral density after use and the relative safety of the drug. All they recommend an exhaustive control of mineral metabolism in the first weeks after administration, in providing calcium and vitamin D and avoid as far as possible concomitant treatment with cinacalcet, especially in mild hyperparathyroidism.

Although the pharmacokinetics and pharmacodynamics of Denosumab seems not influenced by renal function and dose therefore is not to be adjusted in renal insufficiency, they have been described several cases of hypocalcemia in renal patients with varying degrees of impairment of glomerular filtration and renal replacement therapy (1.5-9) [5-8]. It has been reported an increased risk in patients with lower-middle calcemias (as in our case) and treated with hypocalcemic agents such as cinacalcet (9), but has also been described hypocalcemia in patients with medium-high calcemias without concomitant hypocalcemic therapy (1.5-8).

The duration of hypocalcemia is variable according to studies published, but usually days, unless a case published 5 months attributed to co-treatment with cinacalcet (9), unlike our case. Hyperparathyroidism appears to be late more in be correcting in almost the notified patients, but in our ends returning to basal levels. There are few cases and very heterogeneous ones to establish etiologic relationship [9].

\section{Conclusion}

Renal patients rarely included in most clinical pharmacology trials, so nephrologists sometimes treat under attended consent to treat several diseases. Such is the use of Denosumab for osteoporosis in patients with / without renal osteodystrophy. According to few studies, it appears to be effective for the treatment of demineralization, but safer for patients with moderate to severe hyperparathyroidism and calcium figures pre-treatment means should ensure special attention in patients on concomitant treatment with cinacalcet. 


\section{REFERENCES}

1. Sirvent AE, Enriquez R, Sánchez. Hypocalcemia and secondary hyperparathyroidism following Denosumab. Is it safe this drug in chronic kidney disease? Nephrology. 34, 542-544 (2014).

2. Sulkova SD, Horacek J, Safranek R. Denosumab associated with bone density increase and clinical improvement in a longterm hemodialysis patient. Case report and review of the literature. Acta. Médica. 57, 30 34 (2014).

3. Block G, Bone HG, Fang L. A single-dose study of Denosumab in patients with various degrees of renal impairment. J. Bone. Miner. Res. 27, 1471-9 (2012).

4. Chen CL, Chen NC, Hsu CY. An open-label, prospective pilot clinical study of Denosumab for severe hyperparathyroidism in patients with low bone mass undergoing dialysis. J. Clin. Endocrinol. Metab. 99, 2426-32 (2014).

5. Martin-Baez IM, White-Garcia R, M AlonsoSuarez. Posdenosumab severe hypocalcemia. Nephrology. 33, 614-5 (2013).

6. Ungprasert P, Cheungpasitporn W, Srivali N. Life-threating hypocalcemia associated with Denosumab in a patient with moderate renal insufficiency. Am. J. Emerg. Med. 31:756e1-
$756 \mathrm{e} 2$ (2013).

7. McCormick B, Davis J, Burns KD. Severe hypocalcemia following Denosumab injection in a hemodialysis patient. Am. J. Kidney. Dis. 60, 626-628 (2012).

8. Torregrosa JV. Dramatic increase in parathyroid hormone an hypocalcemia after Denosumab in a kidney transplanted patient. Clin. Kidney. J. 6, $122(2012)$.

9. Ivanov P, Khedr M. Prolonged hypocalcaemia following single doses of $60 \mathrm{mg}$ Denosumab in two patients with CKD $4 / 5$ on cinacalcet treatment for tertiary hyperparathyroidism. Clin. Kidney. J. 6, 245 (2013). 